\title{
Prevalence and Precipitating Factors of Migraine in Secondary School Students in Thailand
}

\author{
Vitchayaporn Emarach Saengow ${ }^{1}$ Sirikarn Tangcheewinsirikul ${ }^{1}$ \\ ${ }^{1}$ Department of Pediatrics, Maharat Nakhon Ratchasima Hospital, \\ Nakhon Ratchasima, Thailand \\ J Child Sci 2018;8:e50-e54.

\begin{abstract}
Address for correspondence Vitchayaporn Emarach Saengow, MD Department of Pediatrics, Maharat Nakhon Ratchasima Hospital, Chang Peuk Road, Muang, Nakhon Ratchasima 30000, Thailand (e-mail: vitchayaporn.sa@cpird.in.th).
\end{abstract}

\begin{abstract}
Introduction Migraine is the most common primary headache in children which leads to disturbance of school performance and impaired quality of life. The prevalence of migraine is different between countries and ethnicities; this study determines the prevalence and precipitating factors of migraine in Thailand.

Method This cross-sectional school-based study was conducted in Nakhon Ratchasima. The children aged between 12 and 18 years from four secondary schools were selected by stratified randomization method. The screening questionnaire and directed interview with physical examination were done and migraine was diagnosed based on the criteria of International Classification of Headache Disorders III. The prevalence of migraine was identified and baseline characteristic of disease with precipitating factors was evaluated.

Results A total of 2,744 students aged between 12 and 18 years (900 males, 1,844 females) were recruited; mean age was $14.3 \pm 1.75$ years. Fifty-five students were

Keywords

- migraine

- prevalence

- pediatric

- students

- severity

- precipitating factors diagnosed with migraine (20 males [36.36\%], 35 females [63.64\%], mean age: $15.1 \pm 1.6$ years). The prevalence of migraine was $1.98 \%$. Migraine prevalence was predominant in female adolescents with a common feature being migraine without aura. The important precipitating factor in this study was stress from academic achievement.

Conclusion Migraine is a common disease in children and under-recognized in school students, and stress is the major precipitating factor.
\end{abstract}

\section{Introduction}

Headache, a common health problem, affects almost $75 \%$ of children in their childhood. ${ }^{1}$

Migraine is the most common primary headache which has chronic effects on children's daily activities as well as their quality of life; however, migraine is usually underrecognized by parents, teachers, and physicians. Previous studies revealed that the prevalence of migraine in children is increasing by age: $1.7 \%$ in 7 -year-old children to $5.3 \%$ in 15 year-old children and it also differs in various ethnicities: the prevalence is 2.7 to $22 \%$ in the Western countries and 0.5 to
$13.8 \%$ in Asian countries. ${ }^{1-3}$ The precipitating factors of migraine are different in each study such as emotional stress, menstruation, social expectation, socioeconomic status, and lack of sleep and diet. ${ }^{1-11}$

Previously in Thailand, two studies had looked over the prevalence of migraine in Bangkok, the capital city of Thailand ${ }^{2,3}$ however, there is no survey on prevalence of migraine in other regions with different cultures and environment in Thailand. Therefore, in this study, the prevalence of migraine assessed in children in Northeastern Thailand and the characteristics of migraine and precipitating factors of migraine have been further determined. received

May 26, 2018

accepted after revision

July 31,2018
DOI https://doi.org/

10.1055/s-0038-1669987.

ISSN 2474-5871.
Copyright @ 2018 Georg Thieme Verlag

KG Stuttgart · New York
License terms

(๑) $\odot \circledast$ 


\section{Method}

This cross-sectional school-based study was conducted in Nakhon Ratchasima Province, a town in northeastern Thailand with a total population of 210,941 and 11,175 students aged 12 to 18 years. Following the approval of Institutional Review Board of Maharat Nakhon Ratchasima Hospital, the study was conducted between July 1,2016 and September 30, 2016. Four schools in Mueang Nakhon Ratchasima District with students of grade 7 to 12 were recruited; the children aged between 12 and 18 years were selected by stratified randomization method. The self-administration screening questionnaire was sent to the selected students and 2 weeks apart the screening questionnaires were collected for further evaluations in regard to headache symptoms. Individuals with the most compatible symptoms to migraine were further selected to be examined and interviewed by pediatric neurologists. The diagnosis of migraine and other primary headaches was made based on the diagnostic criteria of International Classification of Headache Disorders III (ICHD-III) as shown in - Table $1 .^{12}$ The baseline characteristic features of migraine (we recruited only cephalic manifestation of migraine), such as frequency, duration, location, quality, intensity of pain, influence of physical activity, nausea, vomiting, photophobia, and phonophobia, the type of migraine, associated signs and symptoms, precipitating factors (diet, stress [evaluated by direct interview], total sleep times, menstruation, social expectation, grade point average [GPA]), and family history, were recorded to determine the prevalence of migraine and precipitating factors. Stata 10 program was used for data analysis. In descriptive analysis, mean \pm standard deviation and percentage were used, while univariate and multivariable analyses were employed to evaluate the associated precipitating factors of migraine by comparing with children without migraine; results are reported as significance levels of $p<0.05$.

Table 1 Diagnosis criteria for migraine without aura (ICHD-III)

\begin{tabular}{|l|}
\hline A. At least five attacks fulfilling criteria B-D \\
\hline $\begin{array}{l}\text { B. Headache attacks lasting 2-72 hours } \\
\text { (untreated or unsuccessfully treated) }\end{array}$ \\
\hline $\begin{array}{l}\text { C. Headache has at least two of the following four } \\
\text { characteristics: }\end{array}$ \\
\hline 1. Unilateral location \\
\hline 2. Pulsating quality \\
\hline 3. Moderate or severe pain intensity \\
\hline $\begin{array}{l}\text { 4. Aggravation or causing avoidance of routine } \\
\text { physical activity (e.g., walking or climbing stairs) }\end{array}$ \\
\hline D. During headache at least one of the following: \\
\hline 1. Nausea and/or vomiting \\
\hline 2. Photophobia and phonophobia \\
\hline E. Not better accounted for by another ICHD-III diagnosis. \\
\hline
\end{tabular}

Abbreviation: ICHD-III, International Classification of Headache Disorders III.
Table 2 Characteristic of migraine

\begin{tabular}{|c|c|}
\hline & $\begin{array}{l}\text { Number of } \\
\text { students (\%) } \\
N=55\end{array}$ \\
\hline \multicolumn{2}{|l|}{ Age } \\
\hline \multicolumn{2}{|l|}{ 12-17 year } \\
\hline \multicolumn{2}{|l|}{ Mean age $15.1 \pm 1.6$ year } \\
\hline \multicolumn{2}{|l|}{ Sex } \\
\hline Male & $20(36.36)$ \\
\hline Female & $35(63.64)$ \\
\hline \multicolumn{2}{|l|}{ Classification of migraine } \\
\hline Migraine without aura & $41(74.54)$ \\
\hline Migraine with typical aura & $12(21.81)$ \\
\hline Hemiplegic migraine & $1(1.81)$ \\
\hline Migraine with brain stem aura & $1(1.81)$ \\
\hline \multicolumn{2}{|l|}{ Characteristic of pain } \\
\hline Throbbing & $33(60.01)$ \\
\hline Tense & $3(5.45)$ \\
\hline Burning & $3(5.45)$ \\
\hline Others & $16(29.09)$ \\
\hline \multicolumn{2}{|l|}{ Duration of headache } \\
\hline 2-4 hours & $38(69.09)$ \\
\hline $4-8$ hours & $13(23.63)$ \\
\hline$>8$ hours & $4(7.27)$ \\
\hline \multicolumn{2}{|l|}{ Site of headache } \\
\hline \multicolumn{2}{|l|}{ Unilateral } \\
\hline Frontal & $1(1.81)$ \\
\hline Temporal & $16(29.09)$ \\
\hline Parietal & $2(3.63)$ \\
\hline \multicolumn{2}{|l|}{ Bilateral } \\
\hline Frontal & $7(12.7)$ \\
\hline Temporal & $15(27.27)$ \\
\hline Others & $14(25.45)$ \\
\hline \multicolumn{2}{|l|}{ Nausea and vomiting } \\
\hline Yes & $55(100.00)$ \\
\hline No & $0(0.00)$ \\
\hline \multicolumn{2}{|l|}{ Photophobia or phonophobia } \\
\hline Yes & $53(96.36 \%)$ \\
\hline No & $2(3.64)$ \\
\hline \multicolumn{2}{|l|}{ Frequency of headache } \\
\hline $1-2$ times/month & $22(40.00)$ \\
\hline 3-4 times/month & $13(23.63)$ \\
\hline$>4$ times/month & $9(16.36)$ \\
\hline Yearly & $11(20.00)$ \\
\hline \multicolumn{2}{|l|}{ Severity of migraine } \\
\hline Mild & $32(58.18)$ \\
\hline Moderate & $21(38.18)$ \\
\hline Severe & $2(3.63)$ \\
\hline
\end{tabular}




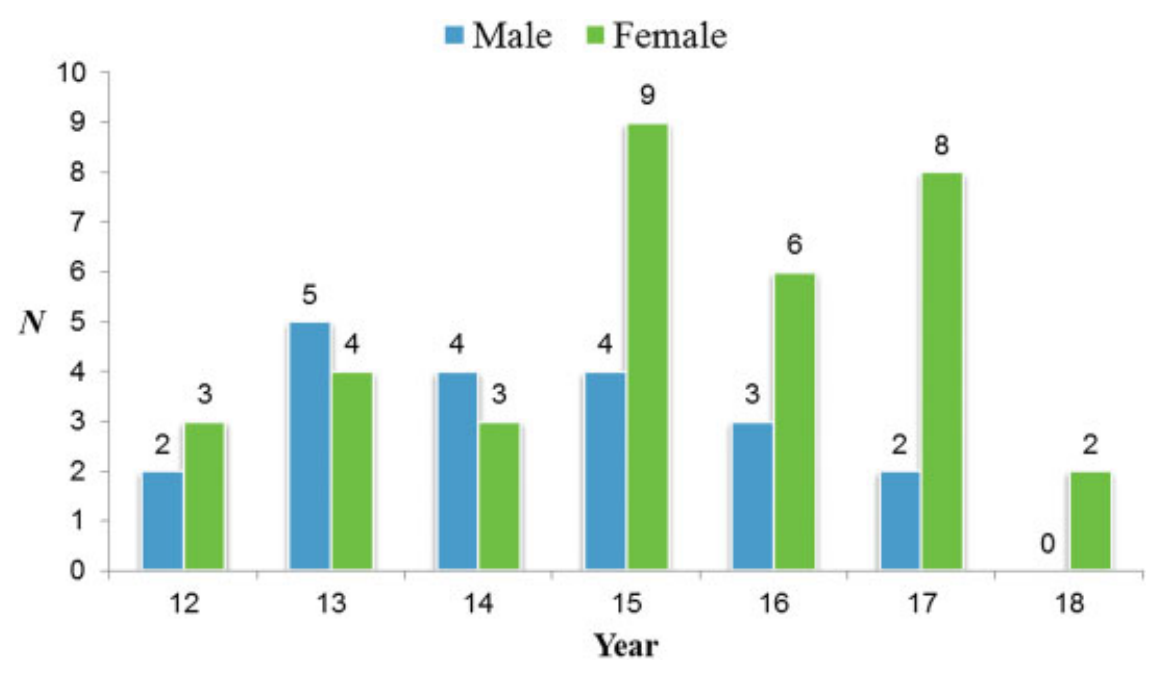

Fig. 1 Age and sex distribution in students with migraine.

\section{Results}

A total of 2,744 students (900 males and 1,844 females) were recruited for this survey; 248 students (91 males [36.69\%], 157 females [63.31\%]), aged between 12 and 18 years (mean age: $14.3 \pm 1.75$ years) were selected using the screening questionnaire. The selected individuals were included into a directed interview and a complete physical examination by a pediatric neurologist. Fifty-five students were diagnosed with migraine in our data (20 males [36.36\%], 35 females [63.64\%], and mean age $15.1 \pm 1.6$ years). The prevalence of migraine was evaluated to be $1.98 \%$. Regarding the characteristic of migraine as shown in - Table 2, commonly found type was migraine without aura (74.54\%); there were two students diagnosed with hemiplegic migraine and migraine with brain stem aura subsequently. Out of all students, $60.61 \%$ had pain characteristics of throbbing or pulsatile, $69.09 \%$ had symptom duration for approximately 2 to 4 hours. The most common site of pain was unilateral temporal region, and the severity of pain was moderate to severe in $98.18 \%$ of students, and
$80 \%$ of students had frequent headaches with one to four migraine attacks per month that affected their quality of life with high impact on their school scores. The mostly affected age group with migraine consisted of students in grade 10 (aged 15 years); in older age, the increase in prevalence happened to be correlated with female sex (as shown in -Fig. 1). The common onset of headache was at the age of 10 years (as shown in - Fig. 2). The family history of migraine was positive in $87.27 \%$ of students, and $7.28 \%$ had comorbidity of obesity and allergic rhinitis. Regarding the lifestyle of students with migraine, $27.27 \%$ of students with migraine enjoyed reading comic books, $25.45 \%$ meditated, and $64.46 \%$ were users of social media and tablets (25 hours per day). About $45.45 \%$ possessed high socioeconomic status (based on family incomes) status and $96.37 \%$ had high value and expectations from family and society. The common range of GPA was 3.00 to 3.5 (34.55\%), and $49.09 \%$ of students diagnosed with migraine did not have any chocolate, green tea, or tea in their daily diet.

Univariate analysis showed that associated precipitating factors of migraine were lack of sleep ( $<6$ hours/day),

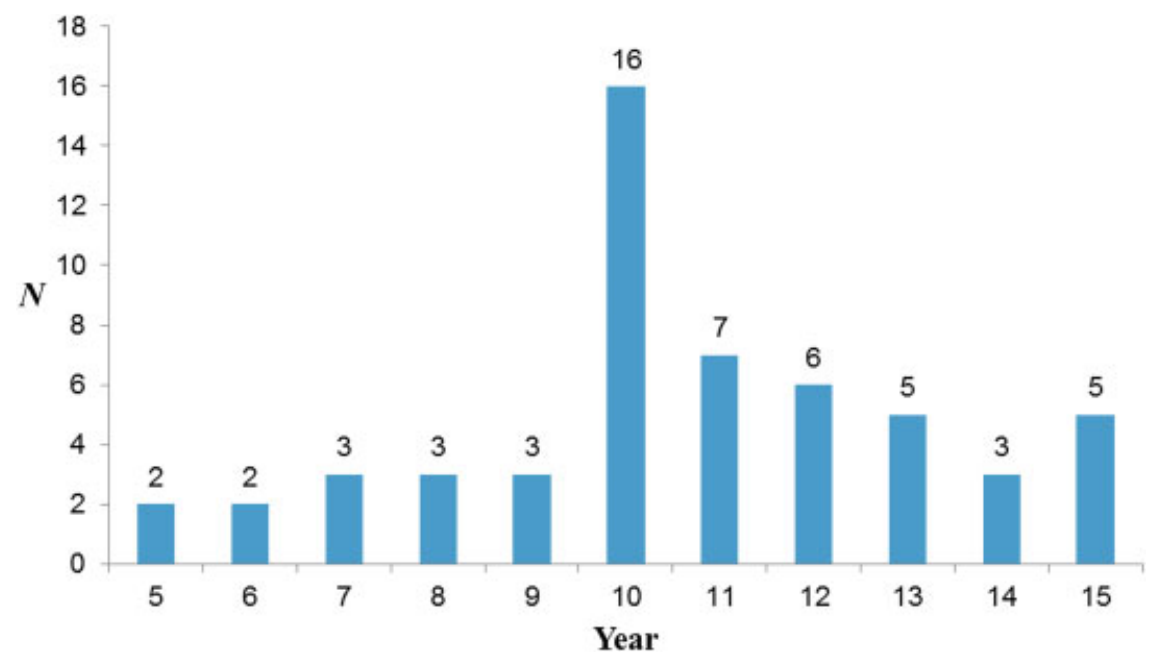

Fig. 2 Age of onset of headache in student with migraine. 
Table 3 Precipitating factors of migraine in univariate analysis

\begin{tabular}{|c|c|c|c|}
\hline Factors & $\begin{array}{l}\text { Migraine } \\
\text { No. (\%) }\end{array}$ & $\begin{array}{l}\text { Not Migraine } \\
\text { No. (\%) }\end{array}$ & $p$-Value \\
\hline \multicolumn{3}{|l|}{ Sleep times } & \multirow[t]{6}{*}{0.03} \\
\hline$<4$ hours & $11(20.0)$ & $26(13.5)$ & \\
\hline 4-6 hours & $22(40.0)$ & $40(20.7)$ & \\
\hline $6-8$ hours & $9(16.4)$ & 75 (38.9) & \\
\hline$>8$ hours & $1(1.8)$ & $6(3.1)$ & \\
\hline $\mathrm{N} / \mathrm{A}$ & $12(21.8)$ & $46(23.8)$ & \\
\hline \multicolumn{3}{|l|}{ Stress } & \multirow[t]{6}{*}{$<0.001$} \\
\hline Family condition & $8(14.5)$ & $28(14.5)$ & \\
\hline $\begin{array}{l}\text { Academic } \\
\text { achievement }\end{array}$ & $27(49.1)$ & $60(31.1)$ & \\
\hline Peer relations & $3(5.5)$ & $7(3.6)$ & \\
\hline Mixed & $13(23.6)$ & $19(9.9)$ & \\
\hline No stress & $4(7.3)$ & $79(40.9)$ & \\
\hline $\begin{array}{l}\text { Precipitated } \\
\text { during } \\
\text { menstruation } \\
\text { period (Female) }\end{array}$ & & & \multirow[t]{3}{*}{0.04} \\
\hline Yes & $13(37.1)$ & 17 (13.9) & \\
\hline No & $22(62.9)$ & $105(86.1)$ & \\
\hline
\end{tabular}

Abbreviation: N/A, not applicable.

academic achievements stress (subject of study, study environment), and menstruation. However, the multivariate analysis showed that associated factor was only the stress from school, while sleeping time and menstruation were considered not significant (-Tables $\mathbf{3}$ and $\mathbf{4}$ ).

\section{Discussion}

A previous study in Asian countries showed the migraine prevalence to be approximately 0.5 to $13.8 \%$; our study reported $1.98 \%$ prevalence of migraine among 2,744 students in Nakhon Ratchasima Province, Thailand, which is lower than the study of Visudtibhan et al in Thailand with the prevalence of $13.8 \%$ among 1,789 students aged between 12 and 15 years living in Bangkok. 2,3 The noticeable difference between these studies can be explained by the divergent culture and social

Table 4 Precipitating factors of migraine in multivariate analysis

\begin{tabular}{|l|l|l|l|}
\hline Factors & $\begin{array}{l}\text { Migraine } \\
\text { No. (\%) }\end{array}$ & $\begin{array}{l}\text { Not Migraine } \\
\text { No. (\%) }\end{array}$ & \multirow{2}{*}{$<0.001$} \\
\hline Stress & & $28(14.5)$ & \\
\cline { 1 - 3 } Family condition & $8(14.5)$ & $60(31.1)$ & \\
\cline { 1 - 3 } $\begin{array}{l}\text { Academic } \\
\text { achievement }\end{array}$ & $27(49.1)$ & $7(3.6)$ & \\
\hline Peer relations & $3(5.5)$ & $19(9.9)$ & \\
\hline Mixed & $13(23.6)$ & $79(40.9)$ & \\
\hline No stress & $4(7.3)$ & 7 & \\
\hline
\end{tabular}

expectations in urban and rural areas of Thailand, which is in agreement with previous study in Taiwan indicating the role of culture in prevalence of migraine. $^{5}$

Studies in Western countries showed that the migraine prevalence ranges from 3 to10.6\% (e.g., in UK [10.6\%], in Italy [3.0\%], in Norway [7\%], and in Turkey [8.8\%]). All previous studies in Western countries showed a higher prevalence of migraine than the prevalence reported in our study which correlates with previous reports indicating lower prevalence of migraine in Asia than Western countries due to the different ethnicity and genetics. 1,6-11 $^{1}$

Even though all studies used different diagnostic criteria for migraine such as International Headache Society, ICHD-II, and ICHD-III classifications, which make a different cut point duration of migraine between 1 and 2 hours, large number of studies had mentioned that the different duration of headache may not have a significant impact on the prevalence of migraine. Similarly, when we compared the ICHD-II (prevalence 2.2\%) and ICHD-III (prevalence 1.98\%), the prevalence analysis did not show a significant difference. However, a study in Japan mentioned the underdiagnoses of migraine if using longer time cutoff. ${ }^{13}$ Further study is required to identify the prevalence of migraine using ICHD-III for a more precise conclusion.

The characteristic of headache such as age and sex distribution of migraine in our survey correlated with other previous studies; female group predominates, especially in older age, with significant higher prevalence. ${ }^{1,5,14-16}$ This study is also in general agreement with previous studies in identifying the strong impact of positive family history associated with migraine as the identification of abnormal genetic in specific type of migraine such as familial hemiplegic migraine. ${ }^{1}$ The migraine pain in this study considered a moderate-to-severe throbbing pain on unilateral site along with nausea and vomiting symptoms as already explained in previous studies. $^{1-3}$

The precipitating factor of migraine in this study was stress from academic achievement which is similar to the previous study in Thailand, ${ }^{2,3}$ while the social expectation was not a significant precipitating factor in this study which illustrates the lower life expectation in rural area than the urban area in Thailand. Similar to other previous studies in Hong Kong and Japan, ${ }^{13,17}$ our data showed stress to be the important precipitating factor in migraine. The sleep deprivation was a significant precipitating factor in Hong Kong and Japan and menstruation was the significant factor in the study of Japan. However, in this study, using the multivariate analysis, the sleep deprivation and menstruation period were not significant. This may be explained by the smaller sample size. Unlike the studies in Western countries, diet was not a precipitating factor of migraine similar to other studies in Thailand and Asia with low impact of food regime on headaches. ${ }^{1}$

\section{Conclusion}

The prevalence of migraine differs between culture and ethnics, with a lower prevalence in Asian and rural areas. Stress is one of the important precipitating factors in Thai and Asian population. 


\section{Acknowledgment}

We would like to thank the staff of Suranaree Wittaya, Ratchasima, Assumption and Marie Vittaya schools for their contribution in facilitating the sampling process. We would also like to thank Professor Anannit Visudtibhan from Ramathibodi Hospital, Mahidol University, Bangkok, Thailand, for his kind suggestions on preparing the screening questionnaires for this study. We are also thankful to Professor Tayard Desudchit from Chulalongkorn University, Bangkok, Thailand for commenting on this survey and its procedure.

\section{References}

1 Hershey AD. Current approaches to the diagnosis and management of paediatric migraine. Lancet Neurol 2010;9(02):190-204

2 Rasmussen BK, Jensen R, Schroll M, Olesen J. Epidemiology of headache in a general population-a prevalence study. J Clin Epidemiol 1991;44(11):1147-1157

3 Visudtibhan A, Thampratankul L, Khongkhatithum C, et al. Migraine in junior high-school students: a prospective 3-academic-year cohort study. Brain Dev 2010;32(10):855-862

4 Visudtibhan A, Siripornpanich V, Khongkhatithum C, et al. Migraine in Thai children: prevalence in junior high school students. J Child Neurol 2007;22(09):1117-1120

5 Wang SJ, Fuh JL, Juang KD, Lu SR. Rising prevalence of migraine in Taiwanese adolescents aged 13-15 years. Cephalalgia 2005;25 (06):433-438

6 Stewart WF, Roy J, Lipton RB. Migraine prevalence, socioeconomic status, and social causation. Neurology 2013;81(11):948-955

7 Split W, Neuman W. Epidemiology of migraine among students from randomly selected secondary schools in Lodz. Headache 1999;39(07):494-501
8 Cavestro C, Montrucchio F, Benci P, et al. Headache prevalence and related symptoms, family history, and treatment habits in a representative population of children in Alba, Italy. Pediatr Neurol 2014;51(03):348-353

9 Milovanović M, Jarebinski M, Martinović Z. Prevalence of primary headaches in children from Belgrade, Serbia. Eur J Paediatr Neurol 2007;11(03):136-141

10 Germany. A nationwide survey of a representative sample on the basis of the headache classification of the International Headache Society. In: Olesen J, ed. Headache Classification and Epidemiology. New York, NY: Raven Press; 1994: 255-261

11 Zencir M, Ergin H, Sahiner T, et al. Epidemiology and symptomatology of migraine among school children: Denizli urban area in Turkey. Headache 2004;44(08):780-785

12 Headache Classification Committee of the International Headache Society (IHS). The International Classification of Headache Disorders, 3rd edition (beta version). Cephalalgia 2013;33(09): 629-628

13 Ando N, Fujimoto S, Ishikawa T, et al. Prevalence and features of migraine in Japanese junior high school students aged 12-15 yr. Brain Dev 2007;29(08):482-485

14 Linet MS, Stewart WF, Celentano DD, Ziegler D, Sprecher M. An epidemiologic study of headache among adolescents and young adults. JAMA 1989;261(15):2211-2216

15 Stewart WF, Lipton RB, Celentano DD, Reed ML. Prevalence of migraine headache in the United States. Relation to age, income, race, and other sociodemographic factors. JAMA 1992;267(01): 64-69

16 Abu-Arefeh I, Russell G. Prevalence of headache and migraine in schoolchildren. BMJ 1994;309(6957):765-769

17 Kong CK, Cheng WW, Wong LY. Epidemiology of headache in Hong Kong primary-level schoolchildren: questionnaire study. Hong Kong Med J 2001;7(01):29-33 